\title{
Study of Dermatophytosis in a Tertiary Care Centre in Central India
}

\author{
Nirmal Channe ${ }^{1}$, Supriya S. Tankhiwale ${ }^{2}$ \\ 1, 2 Department of Microbiology, Government Medical College, Nagpur, Maharashtra, India.
}

\section{ABSTRACT}

\section{BACKGROUND}

Mycoses are assuming greater significance both in developed and developing countries particularly due to advent of immunosuppressive drugs and diseases. Dermatophytosis is most common type of cutaneous fungal infections seen in man, though in past few decades non-dermatophytes are also assuming importance. Present study is undertaken to know the pattern of dermatophytosis from our region.

\section{METHODS}

An observational study was conducted on 150 samples of patients with complaints of superficial mycoses. Samples were processed for microscopy on potassium hydroxide (KOH) mount and culture on Sabouraud's dextrose agar (SDA) with and without cycloheximide and chloramphenicol. Any growth was identified by conventional technique.

\section{RESULTS}

One hundred and fifty samples consisting of 86 skin and 64 nails were studied. Most samples were seen in rainy season and males were predominantly affected. Adults from age of 21 - 50 years were most commonly affected. Most common clinical presentation was tinea corporis (70.93\%). In dermatophytic causes, $T$. mentagrophytes (36.21\%), T. tonsurans (27.59\%) followed by T. rubrum (15.52\%) were common isolates. In non-dermatophytic causes, candida spp. followed by aspergillus spp. were commonest isolates.

\section{CONCLUSIONS}

Tinea corporis is found to be the commonest presentation. Along with dermatophytes, non-dermatophytic fungi are also emerging as the cause of superficial mycoses. In non-dermatophytic fungi, candida is the commonest species, which is now a days showing drug resistance; hence, identification of causative agent is important for correct and prompt treatment.

\section{KEY WORDS}

Dermatophytosis, Non-Dermatophytic Fungi, Dermatophytic Fungi
Corresponding Author: Dr. Supriya S. Tankhiwale, Associate Professor, Government Medical College, Nagpur, Maharashtra, India. E-mail: supriyatankhiwale@yahoo.com

DOI: $10.14260 / \mathrm{jemds} / 2021 / 106$

How to Cite This Article:

Channe N, Tankhiwale SS. Study of dermatophytosis in a tertiary care centre in Central India. J Evolution Med Dent Sci 2021;10(08):484-487, DOI: $10.14260 /$ jemds/2021/106

Submission 23-03-2020,

Peer Review 27-12-2020,

Acceptance 04-01-2021,

Published 22-02-2021.

Copyright (C) 2021 JEMDS. This is an open access article distributed under Creative Commons Attribution License [Attribution 4.0 International (CC BY 4.0)] 


\section{BACKGROUND}

Infections caused by fungi are called mycoses. Mycoses are very common in human beings. They are assuming greater significance both in developed and developing countries particularly due to advent of immunosuppressive drugs and diseases. ${ }^{1}$

According to World Health Organization (WHO), the prevalence rate of superficial mycotic infections worldwide has been found to be $20-25 \% .{ }^{2}$ It is more prevalent in tropical and subtropical countries like India where the heat and humidity is high for most part of the year. ${ }^{3}$

The dermatophytes are a group of closely related fungi that have the capacity to invade keratinised tissue (skin, hair and nails) of humans to produce an infection, dermatophytosis, commonly referred as ringworm. Aetiologic agents of the dermatophytosis are classified as, trichophyton, microsporum and epidermophyton. The trichophyton species usually infect the skin, hair and nails. The microsporum species infect the skin and hair not the nails. The epidermophyton species infect the skin as well as nails but not the hair. Dermatophytosis is most common type of cutaneous fungal infections seen in man. ${ }^{4}$

Dermatophytes affect all races. Socioeconomic factors which result in overcrowding, poor hygiene, malnutrition plays the important role. ${ }^{5}$ However, over the past decades, non-dermatophytes, as agents of superficial fungal infection in humans, produce lesions that are clinically similar to those caused by dermatophytic infections. ${ }^{6}$

This presentation, though very typical of ringworm infection, is often confused with the other skin disorders, making laboratory confirmation necessary for the correct and prompt treatment. However, dermatophyte infections are poorly documented in literature, possibly because of relatively low morbidity and no mortality. ${ }^{7}$ Hence, this study is undertaken to find out the pattern of etiological agents from the region.

\section{METHODS}

This is an observational study conducted from April 2016 to April 2017. A total of 150 samples of patients attending outpatient department (OPD) of dermatology, venereology and leprology department of our institute with complaint of superficial mycosis were included in this study.

Before collecting the samples from skin and nails, relevant history was taken viz; age, sex, occupation and socioeconomic status. Details of clinical lesions such as origin, site, size, colour, itching, any treatment received were noted down. The lesion area was cleaned with $70 \%$ alcohol before sampling to remove contaminants such as bacteria. Specimen were subjected to $10 \%$ potassium hydroxide mount and examined for the presence of fungal hyphae and spores. Two Sabouraud's dextrose agar slopes with and without cycloheximide and chloramphenicol were used for culture of specimens and incubated at temperatures of $25^{\circ} \mathrm{C}$ and $37^{\circ} \mathrm{C}$ for 3 weeks for isolation of dermatophytic and nondermatophytic moulds and yeasts. Any fungal growth was identified based on colony morphology, pigmentation, growth rate, microscopy using lactophenol cotton blue mount (LPCB), slide culture on corn meal agar, urease test, etc. as per conventional techniques. For candida speciation, germ tube test, slide culture and hi-chrome candida chromogenic agar were used. ${ }^{8}$

\section{RESULTS}

A total of one hundred and fifty samples were processed which consists of 86 (57.33\%) skin and 64 (42.67\%) nail samples. Maximum incidence of cases was recorded in rainy season i.e. 57 / 150 (38 \%) followed by summer i.e. 54 / 150 (36 \%) and least in winter season i.e. 39 / 150 (26\%).

Most of the patients belonged to the age group of $21-50$ years i.e. 103 / 150 (68.66 \%). In the age group of 6 month to 20 years, 27 / 150 (18\%) patients were seen and that of 51 80 years, only $20 / 150$ (13.33\%) patients were found.

Among skin, tinea corporis (70.93\%) was the commonest clinical presentation, followed by tinea cruris $(20.93 \%)$ and tinea capitis (4.65\%). (Table 1) Males were 93 / 150 (62\%) and females were 57 / 150 (38\%) with male to female ratio of $1.63: 1$.

\begin{tabular}{|cc|}
\hline Clinical Presentation & Number of Cases \\
Tinea corporis & 61 \\
Tinea cruris & 18 \\
Tinea capitis & 4 \\
Tinea barbae & 2 \\
Tinea pedis & 1 \\
Onychomycosis & 64 \\
Total & 150 \\
\hline Table 1. Distribution of Clinical Presentation \\
\hline
\end{tabular}

\begin{tabular}{|cccc|}
\hline Culture & KOH Positive & KOH Negative & Total \\
Culture positive & 86 & 2 & 88 \\
Culture negative & 24 & 38 & 62 \\
\hline Total & 110 & 40 & 150 \\
\hline
\end{tabular}

Table 2. Distribution of Fungi According to $\mathrm{KOH}$ and Culture Positivity

\begin{tabular}{|cc|}
\hline Dermatophyte Species & Numbers (\%) \\
Trichophyton mentagrophytes & $21(36.21)$ \\
Trichophyton tonsurans & $16(27.59)$ \\
Trichophyton rubrum & $9(15.52)$ \\
Trichophyton verrucosum & $6(10.34)$ \\
Epidermophyton floccosum & $3(5.17)$ \\
Trichophyton violaceum & $2(3.45)$ \\
Microsporum ferrugineum & $1(1.72)$ \\
Total & $58(100)$ \\
\hline Table 3. Distribution of Isolated Dermatophytic Species \\
\hline
\end{tabular}

\begin{tabular}{|cc|}
\hline Non-Dermatophyte Species & Numbers (\%) \\
Candida tropicalis & $7(23.33)$ \\
Candida albicans & $3(10)$ \\
Candida glabrata & $1(3.33)$ \\
Aspergillus nidulans & $6(20)$ \\
Aspergillus glaucus & $4(13.33)$ \\
Fusarium spp. & $3(10)$ \\
Alternaria spp. & $2(6.67)$ \\
Exophiala werneckii & $2(6.67)$ \\
Scedosporium apiospermum & $1(3.33)$ \\
Scytalidium dimidiatum & $1(3.33)$ \\
Total & $30(100)$ \\
\hline Table 4. Distribution of Non-Dermatophytic Fungal Isolates
\end{tabular}

Out of 150 specimens, 110 (73.33\%) were positive for fungal hyphae and spores by direct $\mathrm{KOH}$ preparation and 88 (58.66 \%) revealed growth on culture. Two specimen that 
were negative in $\mathrm{KOH}$ examination revealed growth on culture. Twenty-four specimen which were positive by microscopy failed to grow in culture. Both microscopy and culture negative specimen were 38. (25.33\%) (Table 2)

Out of 88 culture positive specimen, $58(65.90 \%)$ isolates belonged to dermatophytes while $30(34.10 \%)$ belonged to non-dermatophytic moulds and yeasts. The most common isolate among dermatophytes was Trichophyton mentagrophytes (36.21 \%) followed by Trichophyton tonsurans (27.59\%). Third most common being Trichophyton rubrum (15.52\%). Other dermatophytes isolated were $T$. verrucosum, E. floccosum, T. violaceum and M. ferrugineum (Table 3)

Among non-dermatophytes, most common isolate was Candida tropicalis (23.33\%) followed by Aspergillus nidulans (20\%). Third most common being Aspergillus glaucus $(13.33$ $\%)$. There were 3 cases each of fusarium spp. and Candida albicans, 2 cases each of Alternaria and Exophiala werneckii and 1 case each of Candida glabrata, Scedosporium apiospermum and Scytalidium dimidiatum. (Table 4)

\section{DISCUSSION}

The present study is undertaken to find out the pattern of etiological agents of dermatophytosis from our region. A total of 150 cases of clinically diagnosed superficial mycoses were studied. All age groups and both the sexes were included in the study. Maximum patients were in the age group of $21-50$ years. Nilekar et al. ${ }^{9}$ and Parimala Prasad et al. ${ }^{10}$ reported similar observations. Lowest incidence was found above 51 years of age in the present study. More incidence in the youth can be attributed to their outdoor working habits as compared to other age groups.

Only superficial mycoses of nails and skin were received during the study period. Hair samples were not received in the present study. The commonest samples were of skin (57.33 $\%)$. Tinea corporis $(70.93 \%)$ was the commonest clinical presentation, followed by tinea cruris (20.93\%) and tinea capitis (4.65\%). This finding is similar to Lakshmanan et al. ${ }^{11}$ and Ravindra Babu P et al. ${ }^{12}$ who also found tinea corporis as the commonest clinical presentation followed by tinea cruris.

The incidence of dermatophytosis was higher in males (62 $\%$ ) as compared to females (38\%) with male to female ratio of 1.63:1. Vignesh D et al. ${ }^{13}$ 2015; Mohan U et al. ${ }^{14} 1997$ and Peerapur et al. ${ }^{15} 2004$ have also reported higher incidence in males. This can be attributed to the reason that males have to work more outdoors or in the farms as compared to females. So, they are more exposed to hot and humid weather and sweating is more among those involved in manual labour.

Maximum incidence of cases was recorded in rainy season (38\%) which was similar to the observations of Walke HR et al. ${ }^{16}$ and Pramodnath et al. ${ }^{17}$ However Dhobale et al. ${ }^{18}$ 2004, reported maximum incidence in April to August.

In the present study, out of 150 specimens, 110 (73.33\%) were positive for fungal hyphae and spores by direct $\mathrm{KOH}$ preparation. Such a high positive rate was also shown by Huda et al. ${ }^{19}(58.33 \%)$, Damle et al. ${ }^{20}(56.88 \%)$ and Chowdhary et al. ${ }^{21}(56.07 \%)$ and $100 \%$ positivity was shown by Kannan et al. ${ }^{22}$ While Patwardhan et al. ${ }^{23}$ showed a low positivity of 37.40 $\%$. Culture was positive in 88 samples (58.66 \%) in the present study. Similar rate of culture positive specimen was also found by Kannan et al.22 However, Patwardhan et al. ${ }^{23}$ (22.81\%), Damle et al..$^{20}(46.30 \%)$ and Chowdhary et al.21 (46.68\%) showed low to medium rates and Huda et al. ${ }^{19}$ (91.66 \%) showed a very high rate.

Aetiological study showed an isolation of nondermatophytes $(34.10 \%)$ in quite a significant number which were similar to findings of Patel et al. ${ }^{6}(33.34 \%)$ and Grover et al.24 (34 \%). Whereas Kannan et al.22 found nondermatophytes in more than fifty percent of cases (51.5\%).

Among 58 isolates of dermatophytes, 50 were from skin and 8 were from nails. Most common isolate from the skin was T. mentagrophytes and from nails was T. tonsurans.

In the present study, T. mentagrophytes obtained from superficial mycoses was $36.21 \%$ followed by T. tonsurans $(27.59 \%)$ and T. rubrum (15.52\%). T. mentagrophytes as the commonest dermatophyte was also reported by Jain et al. ${ }^{25}$ and Bhatia et al. ${ }^{26}$ No Indian study showed T. tonsurans as the $2^{\text {nd }}$ most common dermatophyte except Weitzman et al. ${ }^{27}$ US. Some Indian studies reported T. rubrum to be the commonest dermatophyte followed by T. mentagrophytes. 7,10

We got a higher isolation rate of dermatophytes (65.90\%) similar to Patel et al. ${ }^{6}$ (66.66 \%) and Grover et al. ${ }^{24}$ (66\%). Whereas Sharma et al. ${ }^{28}$ reported $75 \%$. Slightly less isolation rate of dermatophytes was reported by Kannan et al. ${ }^{22}$ (48.5 $\%)$.

In non-dermatophytic isolates, most common were candida spp. (11 / 30, $36.66 \%$ ) followed by aspergillus spp. $(10 / 30,33.33 \%)$ and fusarium spp. (3 / 30, $10 \%)$. Other nondermatophytes isolated were 2 cases each of Alternaria and Exophiala werneckii and 1 case each of Scedosporium apiospermum and Scytalidium dimidiatum. Similar distribution of non-dermatophytic fungal infection was observed by Lakshmanan et al. ${ }^{11}$ Sharma et al. ${ }^{28}$ reported candida spp. as the commonest isolate followed by Trichosporon asahii and fusarium spp. They didn't find aspergillus which was seen in the present study.

Three species of E. floccosum (5.17\%) were isolated from skin mycoses from cases of tinea corporis (1) and tinea cruris (2). Sharma et al. ${ }^{28}$ also reported E. floccosum (10.66 \%) from tinea corporis and tinea cruris. Higher percentage was reported by Prabhu SR et al. ${ }^{29}$ (29.41 \%). Kannan et al. ${ }^{22}$ found 2 cases (3.77\%) of E. floccosum.

Non-dermatophytic isolates were found in larger number in the present study than other studies which shows that there is change in trend of mycoses in skin and nail. It may be due to excessive use of antibiotics, immunosuppressive drugs apart from personal variables like hygiene, occupation etc.

The variable distribution of different dermatophytic and non-dermatophytic species in different studies may be because of geographical variation, different climatic conditions, patients' history of taking treatment, etc.

\section{CONCLUSIONS}

To conclude, tinea corporis is the commonest clinical presentation of superficial mycoses. Dermatophytes are still the commonest cause of superficial mycoses, $T$. mentagrophytes being the commonest in this region. Besides dermatophytes, non-dermatophytic fungi are also emerging as the cause of superficial mycoses, with candida being the 
commonest species. In the era of drug resistance, it is important to identify the causative agents of superficial mycosis for the correct and prompt treatment.

Data sharing statement provided by the authors is available with the full text of this article at jemds.com.

Financial or other competing interests: None.

Disclosure forms provided by the authors are available with the full text of this article at jemds.com.

\section{REFERENCES}

[1] Singh KA, Srivastava KS. A clinic - mycological study on tineapedis at Ranchi. Ind J Venereal Dermatol $1994 ; 60(2)$ :68 - 71.

[2] WHO. Epidemiology and management of common skin diseases in children in developing countries. Geneva: World Health Organization 2005.

[3] Kumar K, Kindo AJ, Kalyani J, et al. Clinico - mycological profile of dermatophytic skin infections in a tertiary care center- a cross sectional study. Sri Ramachandra Journal of Medicine 2007;1(2) : 12 - 5.

[4] Chander J. Dermatophytoses. In: Textbook of medical mycology. $3^{\text {rd }}$ edn. New Delhi: Mehta Publishers 2011:122-3

[5] Rippon JW. Medical mycology: the pathogenic fungi and the pathogenic actinomycetes. In: Dermophytosis and dermatomycosis. $2^{\text {nd }}$ edn. Philadelphia: W B Saunders Co 1982.

[6] Patel P, Mulla S, Patel D, et al. A study of superficial mycosis in South Gujarat region. National Journal of Community Medicine 2010;1(2):85 - 8.

[7] Johnson RA. Dermatophyte infections in human immune deficiency virus (HIV) disease. Jam Acad Derm 2000;43(Suppl 5):S135 - 42.

[8] Padhye AA, Weitzman I. The Dermatophytes. In: Ajello L, Hay RJ, Collier L, et al. eds. Medical mycology: Topley \& Wilson's microbiology and microbial infection. Vol. 4. 9th edn. Great Britain: Arnold Publication 1998:215-25.

[9] Nilekar SL, Kulkarni VL. Dermatophytosis in and around Ambajogai. IOSR Journal of Dental and Medical Sciences (IOSR-JDMS) 2015;14( 10 ):37-41.

[10] Parimala P, Shivananda PG, Shrinivas CR, et al. Dermatophytosis in and around Manipal. Indian J Dermatol Venereol Leprol 1987;53( 4 ):217-8.

[11] Lakshmanan A, Ganeshkumar P, Mohan SR, et al. Epidemiological and clinical pattern of dermatomycoses in rural India. Indian J Med Microbiol 2015;33( 1):S134-6.

[12] Babu PR, Pravin AJS, Deshmukh G, et al. Efficacy and safety of terbinafine $500 \mathrm{mg}$ once daily in patients with dermatophytosis. Indian J Dermatol 2017;62( 4 ):395 - 9.

[13] Vignesh D, Sankar SP, Sudha S. A clinical study of a superficial dermatophytic infection in kanchipuram. Natl J Med Res Yoga Sci 2015;1(2):22 - 4.
[14] Mohan U, Jindal N, Devi P. Dermatophytosis in Amritsar. Indian J Med Microbiol 1997;15(1) :1197-246.

[15] Peerapur BV, Inamdar AC, Pushpa PV. Clinicomycological study of dermatophytosis in Bijapur. Indian J Med Microbiol 2004;2(4) :273 - 4.

[16] Walke HR, Gaikwad AA, Palekar SS. Clinico-mycological profile of dermatophytosis in patients attending dermatology OPD in tertiary care hospital, India. Int J Curr Microbiol App Sci 2014;3(10):432- 40.

[17] Nath P, Agarwal PK. Some observations on mycotic infections in Luknow. Indian J Med Res 1971 ;59(5):67582.

[18] Dhobale M, Kulkarni RD, Powar RM. Study of Tinea corporis in and around Sangli and Miraj with reference to antifungal drug sensitivity. Indian Med Gazzette 1935 :416 - 8 .

[19] Huda MM, Chakraborty N, Bordoloi JNS. A clinicomycological study of superficial mycosis in Upper Assam. Indian J Dermatol Venerol Leprol 1995 ; 61(6) :329-32.

[20] Damle AS, Fule RP, Kaundianya DV, et al. Mycology of cutaneous fungal infections in Ambajogai: a rural area. Indian J Dermatol Venereol Leprol 1986;47(5) :266 - 8.

[21] Chowdhary AS, Deshmukh AB, Bansal MP, et al. Study of dermatophytoses at Aurangabad. Indian Journal of Medical Microbiology 1986;4(3):229 - 32.

[22] Kannan P, Janaki C, Selvi GS. Prevalence of dermatophytes and other fungal agents isolated from clinical samples. Indian J Med Microbiol 2006;24(3):212 - 5.

[23] Patwardhan N, Dave R. Dermatophytosis in and around Aurangabad. Indian Journal of Pathology and Microbiology 1999;42(4):455 - 62.

[24] Grover S, Roy P. Clinico-mycological profile of superficial mycosis in a hospital in North-East India. Med J Armed Forces India 2003;59(2):114 - 6.

[25] Jain S, Sharma V. Clinicomycological study of dermatophytes in Solan. International Journal of Current Microbiology and Applied Sciences 2015;4(12):190 - 3.

[26] Bhatia VK, Sharma PC. Epidemiological studies on dermatophytosis in human patients in Himachal Pradesh, India. Springerplus 2014;3(1):134.

[27] Weitzman I, Chin NX, Kunjukunju N, et al. A survey of dermatophytes isolated from human patients in the United States from 1993 to 1995. J Am Acad Dermatol 1998;39(2 Pt 1):255 - 61.

[28] Sharma N, Sharma A. To study the species profile in dermatomycoses patients in a tertiary care hospital of Western Maharashtra. Int J Sci Res 2016;5(2) :579 - 81.

[29] Prabhu SR, Shetty VH, Shetty NJ, et al. Clinico-mycological study of superficial fungal infections in coastal Karnataka, India. Journal of Evolution of Medical and Dental Sciences 2013;2(44):8638-46. 